\title{
FIXAÇÃO DA COLUNA VERTEBRAL CERVICAL BAIXA ATRAVÉS DE AMARRILHO INTERESPINHOSO E ENXERTO ÓSSEO
}

\author{
EDSON LOPES JR*, JOSÉ ARNALDO MOTTA DE ARRUDA**, CARLOS VINICIUS MOTA MELO***
}

\begin{abstract}
RESUMO - Descrevemos nossa experiência em 32 pacientes submetidos a fixação da coluna vertebral cervical por via posterior através de amarrilho interespinhoso com fios de aço e enxerto ósseo, retirado do ilíaco, no período de janeiro-1996 a maio-1997 no Serviço de Neurocirurgia do Hospital Municipal Dr. José Frota. Os resultados no período de um ano, de acordo com a escala funcional de Frankel, indicam que dos pacientes que no ingresso apresentavam algum grau de lesão neurológica, 11 melhoraram, 1 piorou e 1 faleceu de infecção respiratória. Não houve piora em nenhum daqueles que na admissão apresentavam ausência de lesão neurológica. Mostramos que o método cirúrgico utilizado é eficaz na fusão da coluna cervical após lesão aguda traumática com comprometimento dos ligamentos posteriores, além de ser uma abordagem de baixo custo.
\end{abstract}

PALAVRAS-CHAVES: trauma raqui-medular cervical, fixação espinhal, amarrilho interespinhoso.

\section{Stabilization of cervical spine with interspinous wiring and autologous bone grafting}

ABSTRACT - Thrity two patients with acute cervical spine trauma were treated at Municipal Hospital Instituto Dr. José Frota, in Fortaleza, Ceará, Brazil, from January 1996 to May 1997. We perfomed posterior spinal stabilization with interspinous wiring and autologous bone grafting when the patients with cervical spine trauma presented dislocations and rupture of the posterior ligamentous complex. The results in the period of one year according to Frankel's functional scale indicate that among the patients with some degree of neurological lesion in the entrance, 11 improved, 1 worsened and 1 died of respiratory tract infection. There was not worsening in none of the 12 patients that presented absence of neurological lesion in the admission. Our data emphasize that the method is effective, safe and accessible.

KEY WORDS: cervical spinal injury, spinal stabilization, interspinous wiring.

Poucas enfermidades ou lesões produzem tão devastadores efeitos, na vida ou na qualidade de vida, como a lesão traumática da coluna vertebral cervical. O prognóstico da quadriplegia que persiste após 72 horas do trauma da coluna vertebral cervical é sombrio, já que somente $8 \%$ desses pacientes terão, após um ano da lesão, alguma função motora nos membros inferiores ${ }^{1}$. Relatos desta tão nefasta lesão remontam de 5000 anos nos papiros de Edwin Smith, que descrevem seis casos de trauma da coluna vertebral, quatro dos quais da coluna vertebral cervical ${ }^{2,3}$, em que o deslocamento ou fratura de vértebras do pescoço produziram nas vítimas priapismo, incontinência urinária e esquecimento de ambos os braços e pernas, e foram descritos como não passíveis de tratamento. A coluna vertebral cervical pela sua mobilidade é a que mais habitualmente sofre lesões em toda a coluna vertebral, podendo o espectro desse sofrimento variar desde cervicalgia a tetraplegia e até a morte ${ }^{4,5}$. Tem como principais agentes causais os acidentes automobilísticos, mergulho em água rasa, quedas e acidentes de atividades atléticas como hipismo e outras ${ }^{5}$. Deve haver suspeita de

Serviço de Neurocirurgia, Hospital Municipal Instituto Dr. José Frota (HMIJF) Fortaleza: *Chefe do Serviço de Neurocirurgia do HMIJF; **Chefe do Serviço de Neurocirurgia do Hospital Geral Dr. César Cals; ***Neurocirurgião do HMIJF. Aceite: 31-outubro-1998.

Dr. Edson Lopes Jr. - Rua Canuto de Aguiar 900300 - 60160-120 Fortaleza CE - Brasil. FAX 0852423419.

E-mail: elopes@brhs.com.br. 
lesão da coluna vertebral cervical nos pacientes com lesões traumáticas acima da clavícula, traumas faciais e traumatismo cranioencefálico principalmente se houver perda de consciência (aproximadamente $10 \%$ de todos os casos de lesão traumática da coluna vertebral cervical ${ }^{1}$ ). Por esta razão, todo paciente vítima de trauma deverá ser mantido com imobilização cervical até que exames radiológicos afastem a presença de fraturas ou deslocamentos da coluna vertebral cervical ${ }^{5}$. É sabido que pela manipulação desastrada de pacientes com lesão incompleta da medula, esta poderá transformar-se em lesão completa, ocasionando grandes danos a eles. Na lesão incompleta o paciente tem condições de recuperar $85 \%$ da função ou mais; na lesão medular completa geralmente recuperará um máximo de $15 \%$. A meta no tratamento da lesão da coluna vertebral cervical é inicialmente o reconhecimento do trauma, seguido por imobilização e alinhamento da coluna quando for evidenciado deslocamento ou fratura de vértebras, a fim de que possamos prevenir lesões adicionais; segue-se a descompressão, reconstituição anatômica e fixação da coluna vertebral cervical ${ }^{5}$.

Apresentamos nossa experiência no tratamento cirúrgico das fraturas e deslizamentos agudos da coluna vertebral cervical por trauma através de técnica de uso corrente em quase todos os serviços de neurocirurgia, que além de seu baixo custo é de comprovada eficiência.

\section{CASUÍSTICA E MÉTODO}

Trinta e dois pacientes, 31 do sexo masculino e 1 do sexo feminino, cuja as idades se encontram na Tabela 1 , vítimas de deslocamentos agudos da coluna vertebral cervical por trauma, foram submetidos a tratamento cirúrgico para fixação da coluna vertebral no Serviço de Neurocirurgia do Hospital Municipal Instituto Dr. José Frota no período de janeiro de 1996 a maio de 1997.

A intervenção cirúrgica foi realizada sempre após a fase aguda do trauma. Em 24 pacientes foi realizada fixação com amarrilho interespinhoso e enxerto ósseo autólogo do ilíaco. Em 8 pacientes que apresentavam material discal ou ósseo no canal vertebral, diagnosticado pela tomografia computadorizada da coluna e/ou mielotomografia, decorrente de fratura por explosão com achatamento do corpo vertebral, associada com lesão dos ligamentos posteriores, ou a hérnia discal traumática que pode se seguir ao deslizamento entre duas vértebras com sobreposição

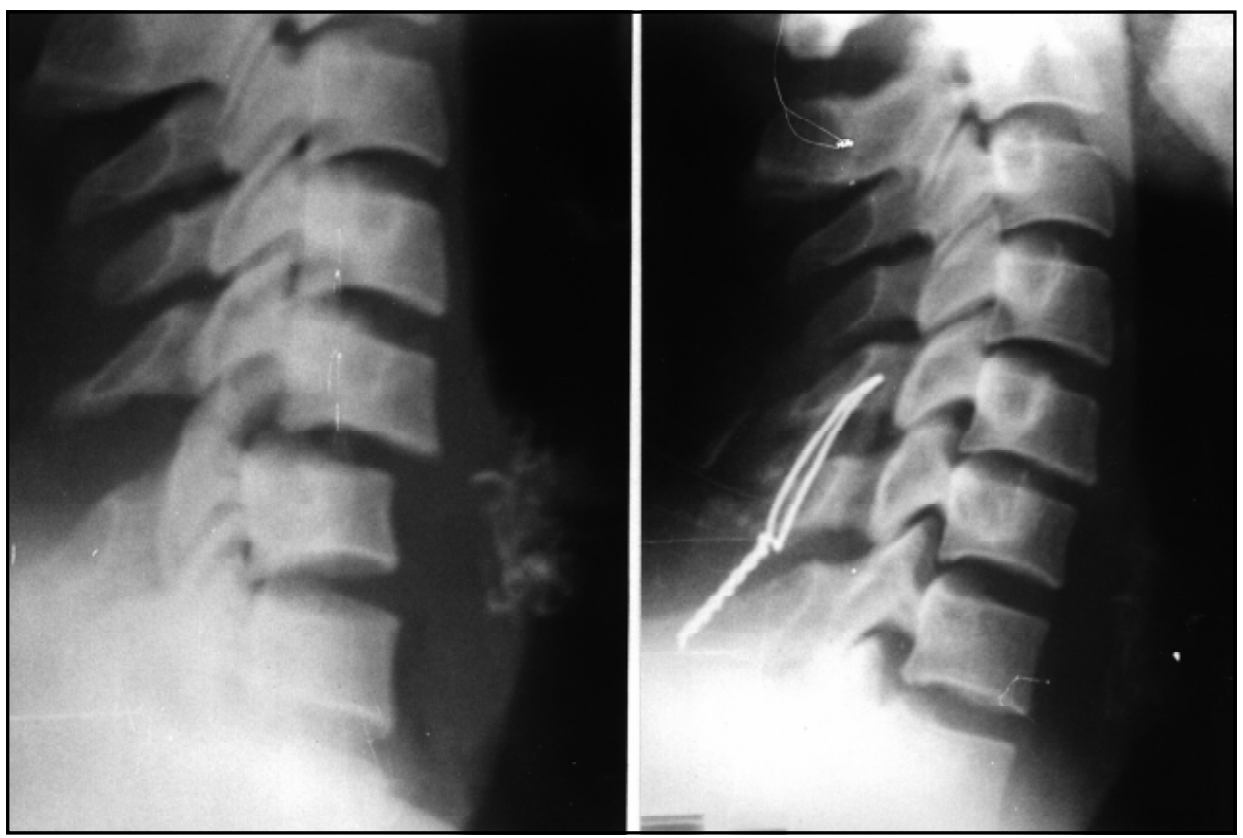

Fig 1. Caso 18. À esquerda, radiografia no pré operatório mostrando deslocamento de C5 sobre C6; paciente em Frankel E. À direita, no pós operatório, radiografia após 6 meses: paciente em Frankel B. 
Tabela 1. Relação Pacientes submetidos a artrodese posterior com amarrilho interespinhoso.

\begin{tabular}{|c|c|c|c|c|c|c|}
\hline Caso & Registro & Sexo & Idade (Anos) & Lesão & Evolução* & Dias de internação \\
\hline 1 & 83992 & M & 22 & C7-T1 & $\mathrm{A}-\mathrm{A}-\mathrm{A}-\mathrm{A}$ & 22 \\
\hline 2 & 84416 & M & 29 & $\mathrm{C} 4-\mathrm{C} 5$ & $E-E-E-E$ & 17 \\
\hline 3 & 82582 & M & 41 & C6-C7 & $\mathrm{A}-\mathrm{A}-\mathrm{A}-\mathrm{A}$ & 27 \\
\hline 4 & 83655 & $\mathrm{M}$ & 48 & $\mathrm{C} 5-\mathrm{C} 6$ & $\mathrm{D}-\mathrm{E}-\mathrm{E}-\mathrm{E}$ & 40 \\
\hline 5 & 85100 & M & 29 & C7-T1 & $A-A-A-A$ & 39 \\
\hline 6 & 86630 & M & 19 & $\mathrm{C} 4-\mathrm{C} 5$ & $E-E-E-E$ & 32 \\
\hline 7 & 87353 & M & 46 & C5-C6 & $D-C-C-C$ & 53 \\
\hline 8 & 88262 & M & 56 & C6-C7 & $E-E-E-E$ & 58 \\
\hline 9 & 90090 & M & 22 & C5-C6 & $E-E-E-E$ & 15 \\
\hline 10 & 84896 & M & 21 & C7-T1 & $A-A-A-A$ & 82 \\
\hline 11 & 85896 & M & 44 & $\mathrm{C} 4-\mathrm{C} 5$ & $\mathrm{~A}-$ Óbito no $7^{\circ} \mathrm{PO}$ & 08 \\
\hline 12 & 90688 & M & 35 & $\mathrm{C} 5-\mathrm{C} 6$ & $\mathrm{D}-\mathrm{E}-\mathrm{E}-\mathrm{E}$ & 28 \\
\hline 13 & u2924/94 & M & 49 & C6-C7 & $\mathrm{D}-\mathrm{E}-\mathrm{E}-\mathrm{E}$ & 08 \\
\hline 14 & 89639 & M & 12 & $\mathrm{C} 3-\mathrm{C} 4$ & $D-E-E-E$ & 12 \\
\hline 15 & 90993 & M & 20 & C6-C7 & $E-E-E-E$ & 21 \\
\hline 16 & 91254 & M & 27 & $\mathrm{C} 5-\mathrm{C} 6$ & $A-B-B-B$ & 28 \\
\hline 17 & 92149 & M & 48 & C5-C6 & $E-E-E-E$ & 12 \\
\hline 18 & 94506 & M & 31 & C5-C6 & $E-E-E-E$ & 13 \\
\hline 19 & 95203 & $\mathrm{M}$ & 58 & C5-C6 & $E-E-E-E$ & 27 \\
\hline 20 & 95375 & M & 16 & $\mathrm{C} 5-\mathrm{C} 6$ & $E-E-E-E$ & 7 \\
\hline 21 & 96232 & $\mathrm{M}$ & 33 & C6-C7 & $A-B-B-B$ & 36 \\
\hline 22 & 96532 & M & 43 & C5-C6 & $A-A-A-A$ & 17 \\
\hline 23 & 95675 & $\mathrm{M}$ & 45 & $\mathrm{C} 3-\mathrm{C} 4$ & $D-E-E-E$ & 14 \\
\hline 24 & 96756 & M & 18 & C6-C7 & $E-E-E-E$ & 15 \\
\hline 25 & 96888 & $\mathrm{M}$ & 41 & C6-C7 & $A-B-B-B$ & 42 \\
\hline 26 & 97991 & $\mathrm{M}$ & 36 & $\mathrm{C} 5-\mathrm{C} 6$ & $\mathrm{~A}-\mathrm{A}-\mathrm{A}-\mathrm{A}$ & 27 \\
\hline 27 & & M & 42 & C6-C7 & $\mathrm{B}-\mathrm{D}-\mathrm{D}-\mathrm{D}$ & 37 \\
\hline 28 & $\mathrm{u} 426 / 96$ & $\mathrm{~F}$ & 55 & $\mathrm{C} 2-\mathrm{C} 3$ & $E-E-E-E$ & 09 \\
\hline 29 & 98162 & M & 46 & C5-C6 & $A-A-A-A$ & 15 \\
\hline 30 & 98321 & M & 43 & C5-C6 & $E-E-E-E$ & 12 \\
\hline 31 & 98691 & M & 18 & C6-C7 & $\mathrm{B}-\mathrm{C}-\mathrm{C}-\mathrm{C}$ & 20 \\
\hline 32 & 99507 & M & 42 & C6-C7 & $D-E-E-E$ & 17 \\
\hline
\end{tabular}

*Evolução de acordo com a escala de Frankel na internação e 3 ,6 e 12 meses após a alta.

Tabela 2. Escala funcional de Frankel.

\begin{tabular}{ll}
\hline Grau & Função Abaixo da Lesão \\
\hline A & Completa ausência de motricidade e de sensação \\
B & Somente a sensação está preservada, ausência completa de motricidade \\
C & Motricidade preservada porém sem função \\
D & Motricidade funcional preservada, podendo apresentar fraqueza ou paralisia dos esfincteres \\
E & Sem lesão \\
\hline
\end{tabular}




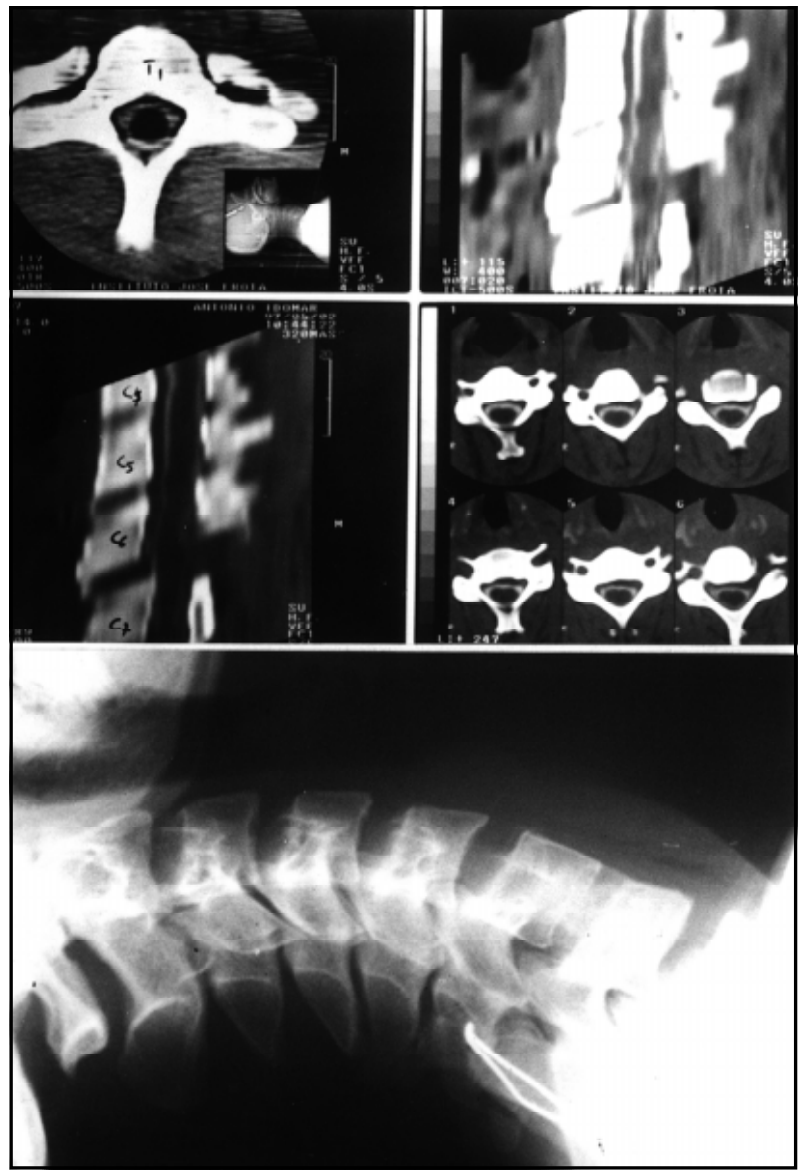

Fig 2. Caso 3. Em cima (A) mielotomografia pré operatória mostrando compressão da medula por fragmentos discais em C6-C7; paciente em Frankel B. Em baixo (B), no pós operatório, radiografia após 10 meses mostrando acesso anterior e posterior, paciente em Frankel $C$.

das facetas articulares ${ }^{6-8}$ foi realizada descompressão por via anterior com enxerto ósseo intersomático, precedida de amarrilho interespinhoso e enxerto ósseo ( abordagem circunferencial) no mesmo tempo cirúrgico.

Todos os pacientes foram avaliados de acordo com a escala funcional de Frankel (Tabela 2) na admissão, 3, 6 e 12 meses após a alta hospitalar. Os agentes causais das lesões são mostrados Tabela 3.

\section{Tabela 3.}

\begin{tabular}{lc}
\hline Causa & $\mathrm{N}^{\mathrm{o}}$ Pacientes \\
\hline Mergulho em água rasa & 13 \\
Acidentes de trânsito & 10 \\
Queda & 7 \\
Agressão & 2 \\
Total & 32 \\
\hline
\end{tabular}

\section{RESULTADOS}

Dos 32 pacientes, 24 apresentavam apenas comprometimento dos ligamentos posteriores sem presença de material discal e/ou fragmentos ósseos no canal raqueano comprimindo a medula cervical e foram submetidos a amarrilho interespinhoso com enxerto ósseo do ilíaco (Fig 1). Nos outros 8 pacientes, que apresentavam compressão da medula cervical em virtude de fragmentos discais ou ósseos, foi realizada descompressão por via 


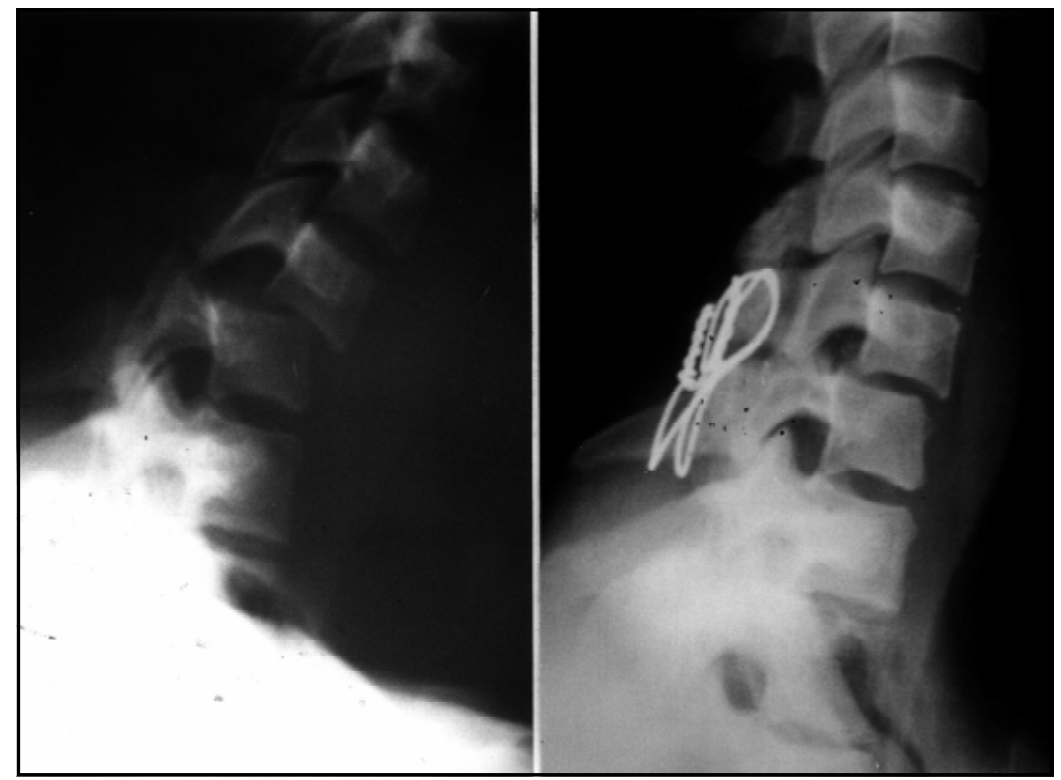

Fig 3. Caso 15. À esquerda, radiografia mostrando luxação C5-C6; paciente em Frankel E. À direita, nos pós operatório, a radiografia após 6 meses da cirurgia não mostrava um perfeito alinhamento mas como o paciente se encontrava em Frankel E e sem deslocamentos nas provas dinâmicas, não foi reoperado.

anterior, com colocação de enxerto ósseo intersomático, seguida de acesso posterior para realização de amarrilho interespinhoso e enxerto ósseo (Fig 2).

Um paciente, com deslizamento de $\mathrm{C} 4$ sobre C5, em grau A na escala funcional de Frankel na admissão, faleceu no pós operatório devido a infecção respiratória. Nos outros 10 pacientes admitidos com quadro clínico de lesão medular completa, 7 permaneceram com ausência completa de motricidade ou sensibilidade abaixo da lesão(grau A na escala de Frankel) e os outros três ascenderam para o nível B. Dois casos foram admitidos em Frankel B e, no seguimento de até 12 meses, um passou a grau $\mathrm{C}$ e outro a grau D. Dos 7 pacientes que na admissão apresentavam-se em grau D de Frankel, 6 ascenderam a E .O único caso de piora no pós operatório imediato era portador de sobreposição das facetas articulares e foi submetido a redução cruenta com fixação por amarrilho interespinhoso. Uma mielotomografia após a cirurgia demonstrou material discal comprimindo a medula, levando-nos a realizar discectomia anterior com enxerto autólogo intersomático. Após um 1 ano e 6 meses do ato cirúrgico, o paciente se encontrava em grau $\mathrm{C}$ de Frankel.

Doze pacientes que foram admitidos com exame neurológico normal permaneceram assim no seguimento de até um ano após a alta hospitalar.

Em três pacientes que apresentavam fraturas da lâmina da vértebra que deslizara, um encontravase em Frankel D e os outros dois em Frankel E. Nestes, realizamos o amarrilho entre os processos espinhosos da vértebra imediatamente superior àquela fraturada ao da vértebra inferior à que fora luxada, obtendo bom alinhamento. Não nos foi possível um perfeito alinhamento em 4 casos, porém como estes encontravam-se em grau E no seguimento de 3, 6 e 12 meses, com provas radiológicas dinâmicas mostrando boa consolidação, não propusemos nova cirurgia, pois havia perfeita artrodese e a função motora e sensitiva estavam preservadas, além de não haver nenhum sintoma radicular (Fig 3).

Todos os pacientes de nossa série foram mantidos com imobilização cervical por colar tipo Philadelphia com proteção de PVC anterior e posterior por período não inferior a 3 meses. Não 
tivemos casos de infecção cirúrgica; usamos antibioticoterapia profilática endovenosa ( $2 \mathrm{~g}$ de oxacilina 1 hora antes de iniciar a incisão cirúrgica e $2 \mathrm{~g}$ de $6 / 6$ horas por 48 horas após o ato cirúrgico) em todos os pacientes. Após receberem alta da sala de recuperação, os pacientes reiniciaram seu tratamento fisioterápico. No terceiro dia do pós operatório, os pacientes que não se encontravam em condições de deambular foram colocados sentados no leito, sempre com o uso de colar cervical. $\mathrm{O}$ período de internação hospitalar, variou de 7 a 92 dias.

\section{DISCUSSÃO}

O tratamento de fraturas e luxações da coluna vertebral tem-se mostrado, na atualidade, muito dispendioso devido ao uso de grande número de próteses (parafusos e placas, entre outros) ${ }^{5,9}$. A técnica cirúrgica que usamos não é novidade, sendo usada na maioria dos serviços de neurocirurgia para o tratamento dos deslizamentos agudos da coluna vertebral cervical. É de comprovada eficácia no tratamento cirúrgico das fraturas instáveis com lesão dos ligamentos posteriores, isto é, movimento de translação do corpo vertebral maior que $3,5 \mathrm{~mm}$ e o ângulo entre dois espaços discais adjacentes maior que 11 graus $^{4,10}$. O amarrilho interespinhoso é técnica relativamente simples, quando comparada com outras formas de instrumentação. Além do seu alto benefício, tem baixo custo e bons resultados.

Ao demonstrar nossa experiência com a técnica apresentada, não temos o desejo de atestar sua superioridade sobre as novas e excitantes técnicas de instrumentação, pois com elas não temos experiência. Contudo, a literatura mostra a inexistência de significativas vantagens clínicas e/ou biomecânicas que elas possam trazer ao tratamento do trauma da coluna vertebral ${ }^{9-12}$, além do desconhecimento dos riscos que podem ocasionar a longo prazo aos pacientes a elas submetidos. A técnica do amarrilho interespinhoso baseia-se na manutenção do alinhamento enquanto se faz a artrodese com osso autólogo, que se consegue por volta de três meses. Durante este período, é mister o uso constante de imobilização cervical.

Em conclusão, nossa experiência mostra que o uso de amarrilho interespinhoso com enxerto ósseo autólogo para fixação e artrodese da coluna vertebral cervical e, quando necessário, associado a descompressão por via anterior com enxerto ósseo intersomático, são métodos eficazes, com baixo índice de complicação e com custo financeiro reduzido ${ }^{5,9}$. Tem, como única desvantagem, o uso constante, por período não inferior a 3 meses, de colar tipo Philadelphia com proteção de PVC anterior e posterior.

\section{REFERÊNCIAS}

1. Maynard FM, Reynolds GG, Fountains S, Wilmot C, Hamilton R. Neurologic prognosis after traumatic quadriplegia. J Neurosurg 1979;50:611-616.

2. Montane I. Historical pespectives of spinal trauma. In Errico TJ, Bauer RD, Waugh T (ed). Spinal trauma. Philadelphia: Linppincott, 1991;1-7.

3. Wagner FC Jr. Injuries to the cervical spine and spinal cord. In Youmans JR, (ed). Neurological surgery. 3.Ed. Philadelphia: Sauders, 1990:2378-2402.

4. Edward C, Benzel M. The anatomical basis of spinal instability. Clin Neurosurg 1993;41:224-241.

5. Lopes E Jr, Arruda JAM, Melo CVM, Parahyba MC. Tratamento cirúrgico de fraturas e deslocamentos agudos da coluna vertebral cervical por trauma. Arq Bras Neurocirurg 1997;16:120-125.

6. Doran SE, Papadoulos SM, Ducker TB, Lillehei KO. Magnetic resonance imaging of coexistent traumatic locked facets of the cervical spine and disc herniation. J Neurosurg 1993;79:341-345.

7. Lintner DM, Knight RQ, Cullen JP. Sequelae of cervical spine facet injuries: the role of canal diameter. Spine 1993;18:725-729.

8. Shapiro SA. Management of unilateral locked facet of the cervical spine. Neurosurgery 1993;33:832-837.

9. Watts C, Smith H, Knoller N. Risks and cost-effectiveness of sublaminar wiring in posterior fusion of cervical spine trauma. Surg. Neurol 1993;40:457-460.

10. Gill K, Paschal S, Corin J, Ashman R, Buchotz RW. A biomechanical comparison of different posterior fusion techniques. Spine 1988 13:813-816.

11. Coe JD, Warden KE, Sutterlin CE, McAfee PC. Biomechanical evaluation of cervical spinal methods in a human cadaveric model. Spine 1989;14:1122-1131.

12. Stephen M, Papadoulos SM, Julian TH. Anatomical treatment of cervical spondilosis. Clin Neurosurg 1993;41:270-285. 